\title{
Do preterm infants always have flattened heads?
}

\author{
N Rutter, W Hinchliffe, P H T Cartlidge
}

\begin{abstract}
It has previously been shown that a soft mattress lessens the degree of head flattening that occurs in preterm infants in the early weeks of life. A follow up study of 31 infants of $<32$ weeks' gestation found head flattening had resolved by 3-4 years of age, regardless of the type of mattress used in the neonatal period, and the children had heads similar in shape to full term controls.
\end{abstract}

(Arch Dis Child 1993; 68: 606-607)

At birth the shape of a baby's head is unrelated to gestation. Amniotic fluid protects the skull from localised deforming forces, so that head shape is determined by unrestrained growth. After birth, the shape of the preterm infant's head changes in comparison with the term infant, becoming increasingly flattened from side to side. This is the result of postnatal moulding, caused by local deforming forces the immature infant lies with the head to one side or the other, is relatively immobile, and the skull bones are thin and soft.

We have shown that in early infancy this head flattening can be reduced if preterm infants are nursed from birth on a soft air filled mattress rather than the conventional firm incubator or cot mattress. ${ }^{1}$ Differences in head shape were present within a few days, and by 3 weeks of age were marked. We carried out the present study to see if these differences persisted into childhood.

\section{Subjects and methods}

Of the 34 original infants who were studied, two had died. One family declined to take part. The remaining 31 were studied by one observer (WH). Fifteen (nine boys, six girls) had been nursed on the conventional firm mattress and 16 (eight boys, eight girls) on the air mattress. Their original gestational age was 26-31 weeks, birth weight 990-1850 g, and their head shape had been monitored until they were 3 weeks old. The 31 infants were restudied at a mean age of $3 \cdot 7$ years (range $3 \cdot 2-4 \cdot 1$ ).

Head shape was assessed in several ways.

Dr Rutter.

Accepted 14 December 1992
Occipitofrontal circumference was measured conventionally with a paper tape. Occipitofrontal and biparietal diameters were measured using an anthropometer (Holton Ltd). The diameters were measured on the line of the occipitofrontal circumference. Occipitofrontal diameter was measured between the most prominent parts of the frontal and occipital bones in the sagittal plane. Biparietal diameter was measured between the two parietal eminences. A ratio of the two was used as an index of head flattening (the higher the ratio, the flatter the head). The inner canthal and outer canthal separation of the eyes was measured using a hand held sliding rule. A flexible draughtsman's curve was placed around the head along the occipitofrontal circumference and the outline traced on to paper. Finally, three standard photos were taken of each child's head: frontal, in profile, and directly from above.

As no control information exists on the head shape of young children born at full term, the occipitofrontal and biparietal diameters and intercanthal distances were similarly measured in 28 children ( 13 boys, 15 girls) born at 38 weeks' gestation or more. These were children at a normal nursery school, mean age 4.0 years (range $3 \cdot 3-4 \cdot 5$ ).

Differences between the two study groups and between each study group and the controls were compared using the Mann-Whitney $U$ test.

\section{Results}

There was no significant difference between the two study groups or between these and the controls with respect to the ratio of occipitofrontal and biparietal diameters, occipitofrontal circumference, and the inner and outer canthal distances (table 1). When the tracings of head shape and the photos were studied by an observer who did not know which study group each child had been in, it was not possible to show that children with rounder or flatter heads originated more frequently from either group.

When the measurements from boys and girls were studied separately, there was again no significant difference in head shape between the groups. However, within each group the mean occipitofrontal to biparietal diameter ratio was higher in boys than girls (table 2). The difference was significant in the control group $(\mathrm{p}<0.01)$.

\section{Discussion}

The difference in head shape between the two study groups of preterm infants that we origi-

nally observed had disappeared by the age of 4
Preterm

Air mattress $(n=16)$

Full term controls $(n=28)$

$\begin{array}{llll}50.6(1.4) & 1.31(0.04) & 27.3(1.4) & 90.3(3.3) \\ 51.0(1.6) & 1.33(0.04) & 27.4(1.8) & 91.1(5.2) \\ 51.8(1.6) & 1.29(0.06) & 28 \cdot 1(1.3) & 91.0(3.6)\end{array}$


Table 2 Sex differences in head shape (expressed as mean occipitofrontal diameter (OFD) to biparietal diameter (BPD) ratio) in the two study groups and the controls

\begin{tabular}{llll}
\hline & \multicolumn{2}{l}{ OFD:BPD ratio } & \\
\cline { 2 - 3 } & Girls & Boys & \\
\hline Preterm & & & \\
Firm mattress & $1.30(n=6)$ & $1.33(n=9)$ & NS \\
Air mattress & $1.32(n=8)$ & $1.34(n=8)$ & NS \\
Full term controls & $1.26(n=15)$ & $1.33(n=13)$ & $p<0.01$ \\
\hline
\end{tabular}

years. At this age the size and shape of their heads was similar to that of control children who were born at term. This suggests that the head flattening commonly seen in preterm infants in early life improves with time, irrespective of the way in which the infant is nursed in the newborn period and of the degree of flattening. In our original study the heads of the preterm infants nursed on a firm mattress were becoming increasingly flatter when last measured at 3 weeks of age (occipitofrontal to biparietal diameter ratio 1.48 compared with 1.40 in those nursed on an air mattress). With the passage of time, growth and remodelling have removed these differences and the overall effect of prematurity. Presumably this takes place after the deforming forces on the immature skull have waned because the infant is able to change head position easily and the skull bones are less pliable.

There is little information on the head shape of ex-preterm infants. Baum and Searls measured the heads of 45 young adults who were born between 1950 and 1953 weighing $1500 \mathrm{~g}$ or less. ${ }^{23}$ They found that the biparietal diameter was lower and the occipitofrontal diameter greater than they expected for their Colorado population. However, the mean occipitofrontal to biparietal diameter ratio was 1.33 , similar to our 4 year old ex-preterm infants and our term controls, suggesting that their adults might not have had abnormally flattened heads. There is even less information on the head shape of children born at term. Our finding that at the age of 4 years girls have significantly more rounded heads than boys was a surprising one.

It is good to know that head flattening in preterm infants resolves with time. We would argue though that this is no justification for not trying to prevent it from happening. The narrow head gives falsely high measurements of occipitofrontal circumference because for a given area the perimeter of an ellipse is greater than that of a circle. This was recognised by Baum and Searls in 1971 but is not widely appreciated. Furthermore, a flattened head with a high narrow forehead and a long narrow face is perceived by parents to be less attractive than the usual rounded head and face of a term infant. ${ }^{4-6}$ The use of a soft, yielding mattress in the early weeks of life effectively prevents this postural deformity.

We are grateful to the staff, parents, and children of the Arboretum Nursery School for their cooperation.

1 Cartlidge PHT, Rutter N. Reduction of head flattening in preterm infants. Arch Dis Child 1988; 63: 755-7.

2 Baum JD, Searls D. Head shape and size of preterm lowbirthweight infants. Dev Med Child Neurol 1971; 13:

3 Baum JD, Searls D. Head shape and size of newborn infants. Dev Med Child Neurol 1971; 13: 572-5.

4 Bull MJ, Bryson CQ, Schreiner RL, Lenono JA. Follow up of infants after intensive care. Perinatology Neonatology 1986; 9: 23-8.

5 Budreau GK. Postnatal cranial moulding and infant attractiveness: implications for nursing. Neonatal Network 1987; 5: 13-9.

6 Budreau GK. The perceived attractiveness of preterm infants with cranial moulding. $f$ Obstet Gynecol Neonatal Nurs 1989; 18: 33-44. 